Flávio Ribeiro ALVES

Pedro Primo

BOMBONATO ${ }^{1}$

Arani Nanci Bomfim

MARIANA $^{1}$

Porfírio Candanedo

GUERRA ${ }^{2}$

Pedro Paulo MACHADO ${ }^{3}$

Carlos Eduardo CRUZ-

PINTO $^{1}$

Antônio Augusto

MACHADO JÚNIOR ${ }^{4}$

Correspondência para:

Faculdade de Medicina Veterinária e ZootecniaFMVZ-USP

Av. Professor Orlando Marques de Paiva, no 87, fone: (11) 3091-7690 CEP: 05508900

flaviovet@usp.br

Recebido para publicação: 25/05/2006 Aprovado para publicação: 17/08/2007

\title{
Anatomia dos tendões e ligamentos da região distal dos membros torácicos de asininos (Equus asinus) utilizados como veículo de tração animal e suas relações com a anatomia do eqüino doméstico
}

\author{
1 - Departamento de Cirurgia da Faculdade de Medicina Veterinária e Zootecnia da \\ Universidade de São Paulo, São Paulo-SP \\ 2 - Departamento das Clínicas do Curso de Medicina Veterinária da Universidade \\ Estadual do Maranhão, São Luis-MA \\ 3 - Médico Veterinário Autônomo \\ 4 - Curso de Medicina Veterinária da Universidade Federal do Piauí, Campos Bom \\ Jesus-PI
}

\section{Resumo}

A espécie asinina (E. asinus), desenvolve um papel fundamental na região Nordeste do Brasil, influenciando diretamente na renda familiar e conseqüentemente na economia local. Tendo em vista o grande número de afecções locomotoras e a falta de cuidados a que estão submetidos, realizou-se um estudo anatômico da região distal de seus membros torácicos, buscando-se subsídios à prática clínica e cirúrgica dedicada a esses animais, bem como a compreensão de sua maior resistência em apresentar sintomatologia frente a lesões locomotoras, quando comparado ao eqüino. A avaliação anatômica revelou características musculares semelhantes às já descritas para eqüinos. Os tendões e ligamentos desses animais apresentaram características particulares, mostrando suas origens e inserções, muitas vezes diferenciados do observado em eqüinos, emitindo projeções tendíneas que permitem uma melhor inserção articular e maior estabilidade do aparelho suspensório, garantindo mais segurança de movimentos durante as atividades físicas.

\section{Introdução}

Em 1967 foi encontrado um esqueleto numa rocha da época eocena no sul dos Estados Unidos. ${ }^{1}$ Tratava-se de um exemplar do Equus ohippus, antepassado mais próximo do eqüino atual, a partir do qual pode ter ocorrido um desenvolvimento por um período de 60 milhões de anos, até o surgimento do Equus caballus (há cerca de 1 milhão de anos). ${ }^{2}$

O E.ohippus era aproximadamente do tamanho de uma raposa, apresentando quatro dígitos nos membros torácicos, três nos membros pélvicos e sua pelagem era, possivelmente, mosqueada ou listrada para que ele pudesse camuflar-se no seu ambiente. ${ }^{1,2}$
Palavras-chave:

Asininos.

Tendões.

Ligamentos articulares. Falanges de animal.
O jumento doméstico (E. asinus vulgaris) provavelmente se originou do mesmo tronco filogenético que deu origem ao cavalo. ${ }^{2,3,4}$ Dados paleontológicos remontam fósseis antigos desses animais datando da era Terciária, período Pliocênico, descobertos na Ilha Pianosa, no Mar Adriático Meridional. Observou-se ainda a existência de fósseis na região da Argélia, considerada terreno da era Quaternária, onde foram designados como E. asinus atlanticus. ${ }^{1,4}$

Os asininos são dotados de grande resistência física quando comparados aos eqüinos. Contudo, rotineiramente apresentam alterações em seu aparelho locomotor, em especial na região distal de seus membros torácicos, havendo então a necessidade de um conhecimento 
aprofundado das estruturas anatômicas que compõem essa região. ${ }^{2}$

Todas estas estruturas foram amplamente estudadas para os eqüinos. Porém, não há a mesma preocupação por parte dos pesquisadores para com os asininos, que são considerados sem diferenciação dos eqüinos. Por essa razão e por ser o asinino o animal de eleição para trabalho na região Nordeste, esta pesquisa teve como objetivo realizar um estudo anatômico dos tendões e ligamentos da região distal dos membros desses animais, caracterizando suas particularidades ou semelhanças dentro do mesmo gênero, gerando mais subsídios à prática clínica $\mathrm{e}$ cirúrgica destinada a esses animais.

\section{Material e Método}

Durante a fase experimental deste estudo foram analisados os membros torácicos de 15 asininos, entre machos e fêmeas. Os animais pesavam em média 150 $\mathrm{kg}$, foram alimentados em pasto natural, e utilizados em veículos de tração animal, todos submetidos às mesmas condições de trabalho. As peças dos membros, constituídas pelas estruturas existentes abaixo da fileira distal dos ossos do carpo, eram oriundas de animais proveniente da Secretaria de Transportes e Urbanismo do Estado do Maranhão - SEMTURB. Todos os animais do experimento morreram por causas naturais (senilidade), processos patológicos ou ainda acidentes automobilísticos.

As peças foram fixadas em solução de formalina a $10 \%$ por um período mínimo de 48 horas e submetidas à dissecação, que se caracterizou pela individualização dos tendões e seus ventres musculares, bem como dos ligamentos de maior contribuição para a região estudada. Foram realizados cortes longitudinais da região distal dos membros torácicos desses animais, com vistas a auxiliar a identificação dos pontos de origem e inserção dos principais ligamentos e tendões, bem como evidenciar a extensão de suas bolsas articulares.

\section{Resultados}

A musculatura dos membros torácicos dos asininos permitiu evidenciar dois grupamentos musculares específicos, sendo uma divisão extensora e outra flexora.

Em asininos, o músculo extensor digital comum constituiu-se como um músculo de maior volume e possivelmente o principal músculo envolvido no mecanismo de extensão da região distal dos membros torácicos. Apresenta um ventre penado, disposto craniolateralmente e fazendo relação com o músculo extensor carpoulnar mais cranialmente (Figura 1). Seu tendão surge aproximadamente a 5

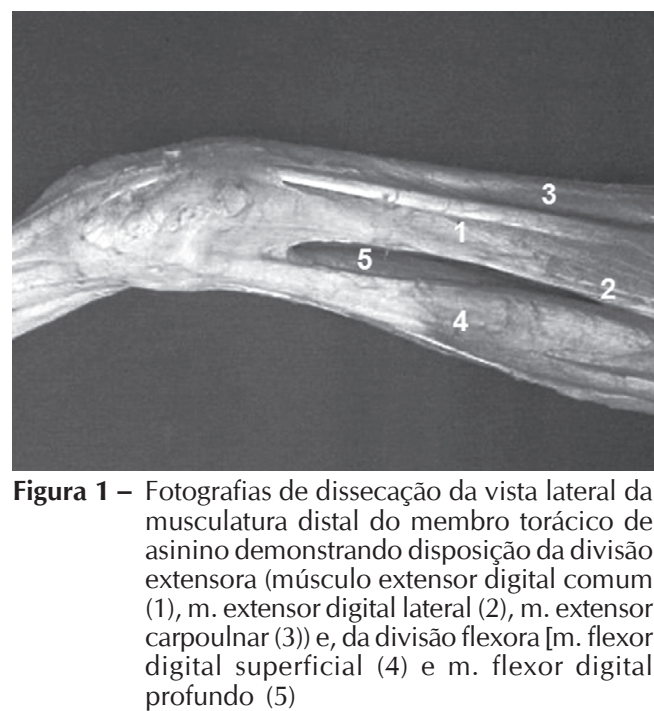

centímetros da epífise distal do rádio, partindo do meio do ventre muscular e seguindo através do sulco extensor do rádio e sobre a cápsula articular cárpica. Passa distalmente sobre a superfície dorsal do terceiro metacarpiano, inclinando-se medialmente, até alcançar a linha média do membro, próximo à articulação metacarpofalangeana, emitindo algumas fibras tendíneas que vão se inserir na extremidade distal do metacarpo (Figura 2). Logo a partir desta, sofre uma dilatação, onde recebe os ramos extensores do ligamento interósseo de ambos os lados (Figura 3 a, b). Alcança sua máxima espessura no ponto onde se insere no processo extensor 


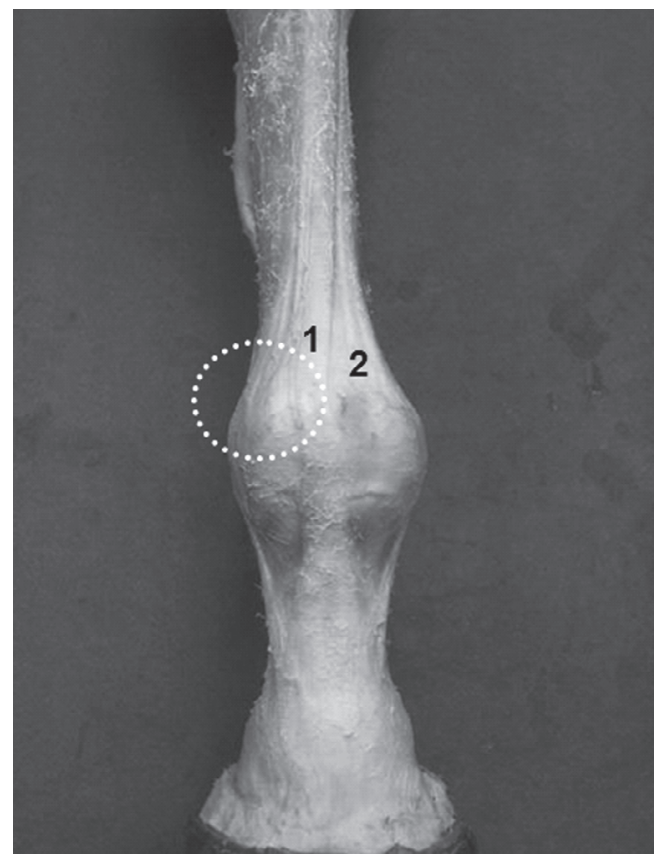

Figura 2 - Fotografia de dissecação da extremidade distal do metacarpo direito de um asinino, demonstrando o ponto de inserção das fibras tendíneas (circulo) do tendão extensor digital comum (1) e o trajeto do tendão extensor digital lateral sobre esse osso (2)
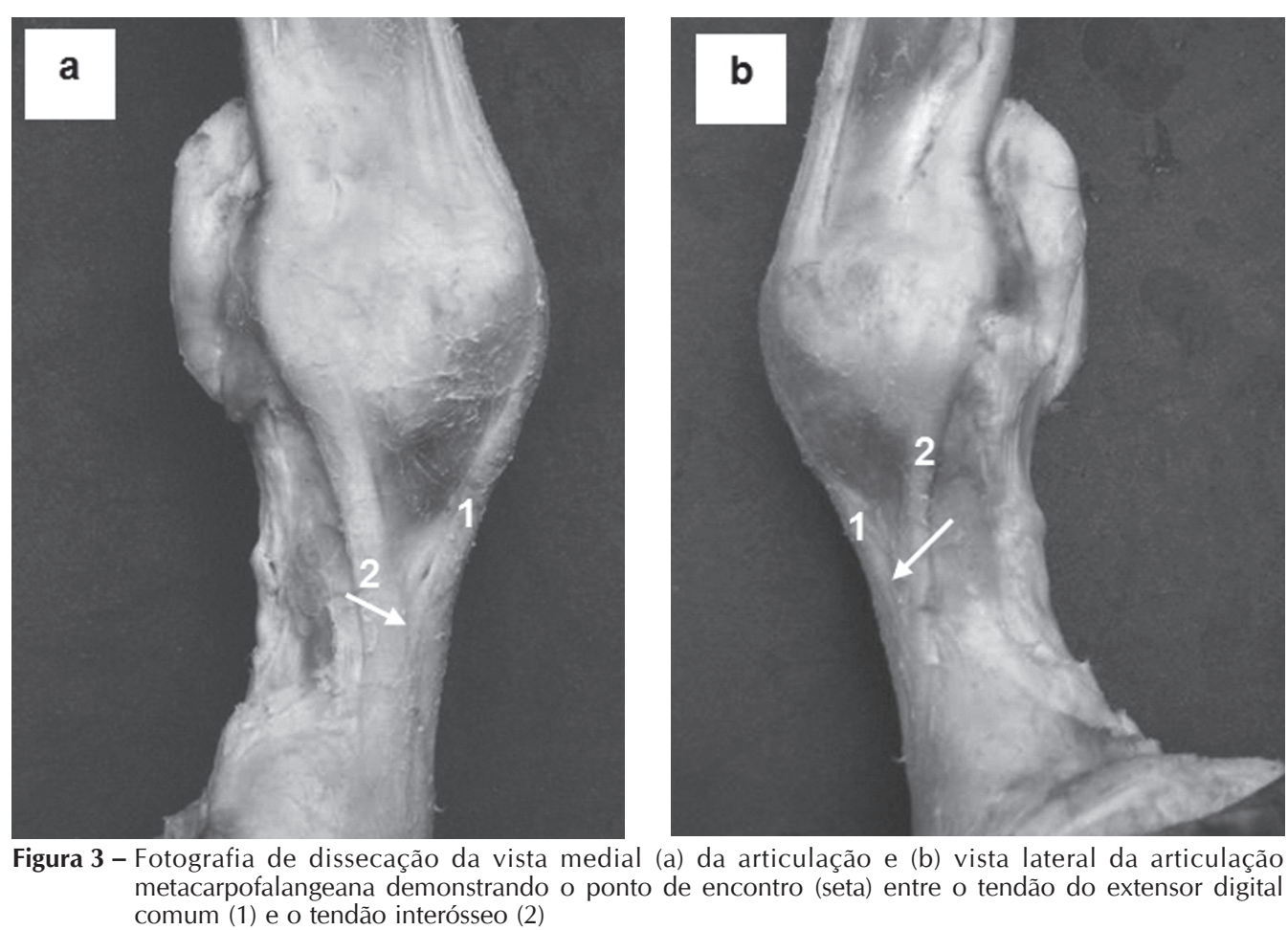

Figura 3 - Fotografia de dissecação da vista medial (a) da articulação e (b) vista lateral da articulação metacarpofalangeana demonstrando o ponto de encontro (seta) entre o tendão do extensor digital comum (1) e o tendão interósseo (2)

da falange distal.

O músculo extensor digital lateral mostra-se duas vezes menor em volume quando comparado ao extensor digital comum. Apresenta um ventre penado e de caráter fusiforme, embora com fibras musculares pouco definidas e que se misturam às do extensor digital comum em sua origem. Seu tendão surge bem próximo à articulação carpiana, quando passa através do sulco do processo estilóide lateral, na extremidade distal do rádio, onde está envolvido pelo retináculo flexor. Orienta-se a partir de uma posição medial, seguindo cada vez mais dorsalmente, porém, não chegando a atingir a linha média do terceiro osso metacarpiano. Durante o seu percurso segue junto ao tendão do músculo extensor digital comum. Próximo à articulação metacarpofalangeana passa a orientar-se com um caráter divergente em relação ao músculo extensor digital comum, emitindo fibras que vão se inserir em uma tuberosidade, na extremidade distal do terceiro osso metacarpiano (Figura 2).

O músculo flexor digital superficial é 
o músculo da divisão flexora mais comumente observado à dissecação anatômica. Seu ventre é multipenado e com fibras musculares que se entremeam às do músculo flexor digital profundo, dificultando uma delimitação definida entre eles. Apresenta um forte tendão que segue distalmente ao nível do canal cárpico junto ao tendão do músculo flexor digital profundo (Figura 1), quando é envolvido pelo retináculo flexor. Distal ao carpo, tornase achatado dorsopalmarmente, adquirindo um caráter elipsóide. Nesse ponto apresentase fortemente aderido à articulação metacarpofalangeana por meio do ligamento anular palmar e mais distalmente pelo ligamento digital palmar proximal. Ao chegar à extremidade distal da falange proximal emite dois ramos que divergem até seus pontos de inserção, nas extremidades proximal e distal da falange média.

O músculo flexor digital profundo (Figura 1) é o segundo músculo da divisão flexora que se estende além da articulação do carpo e que se encontra relacionado com a manutenção do aparelho suspensório em asininos. Apresenta-se constituído por três cabeças: umeral, ulnar e radial, das quais a cabeça umeral fornece a maior contribuição para a execução do trabalho muscular, apresentando um tendão resistente que se une às outras duas, próximo da articulação e passa através do canal cárpico. $\mathrm{O}$ tendão do músculo flexor digital profundo relaciona-se dorsalmente com o ligamento interósseo e na face palmar com o tendão do músculo flexor digital superficial. Passa através do anel do músculo flexor digital superficial até sua inserção na superfície da cartilagem da falange distal, onde forma uma expansão observada entre este tendão e o osso sesamóide distal, conhecida como bolsa podotroclear (Figura 4).

O ligamento interósseo, nos asininos, é bastante desenvolvido. Surge na extremidade proximal do terceiro osso metacarpiano, com suas fibras orientandose longitudinalmente sob a superfície palmar do mesmo e ocupando todo o espaço entre o segundo e quarto ossos metacarpianos.

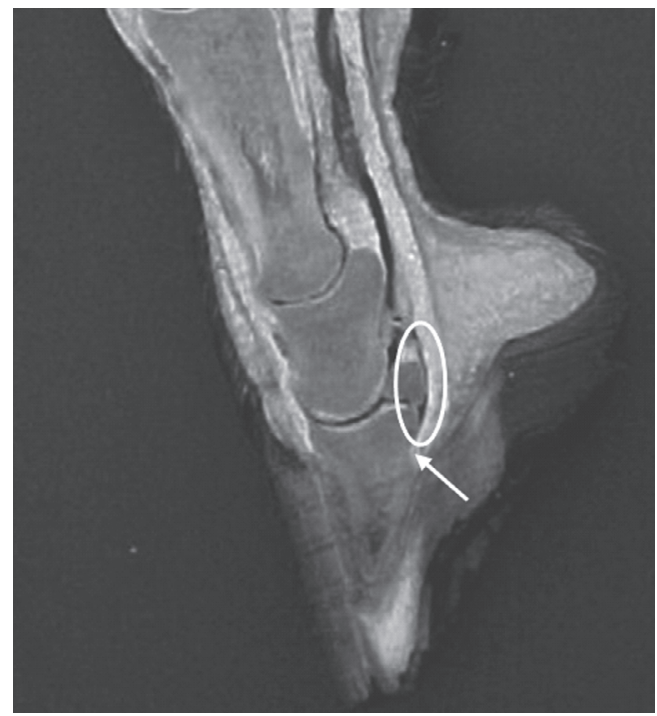

Figura 4 - Corte sagital da região distal do membro torácico de um asinino evidenciando o ponto de inserção do tendão flexor digital profundo (cabeça de seta), e bolsa podotroclear (círculo)

Imediatamente distal ao metacarpo, sofre uma bifurcação, emitindo dois ramos extensores que passam a orientar-se sobre a superfície abaxial dos ossos sesamóides proximais e sobre a cápsula articular (Figura 5). Logo após a articulação metacarpofalangeana, desloca-se mais dorsalmente, até se encontrar com o tendão do músculo extensor digital comum.

Em dissecações mais profundas foi possível evidenciar-se os principais ligamentos que fazem parte do aparelho suspensor nesses animais. O ligamento sesamóideo reto caracterizou-se por fibras que se dispõem em sentido longitudinal, tendo seu ponto de origem em toda extensão da região distal ao ligamento palmar e lateralmente à base dos ossos sesamóides proximais, indo inserir-se distalmente à fibrocartilagem complementar da extremidade proximal da falange média (Figura 6). Os ligamentos sesamóideos oblíquos também apresentam fibras dispostas longitudinalmente, sendo menores em dimensão e extensão quando comparados ao primeiro e apresentam sua inserção proximal ao nível do ligamento palmar e base dos ossos sesamóides proximais. Sua inserção distal encontra-se 


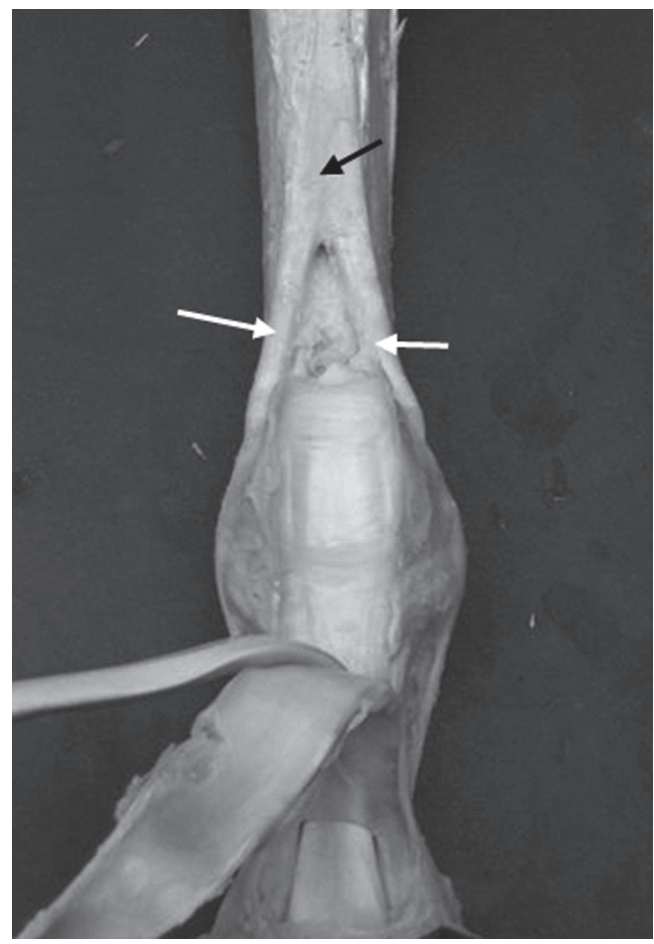

Figura 5 - Fotografia de dissecação da vista palmar do membro torácico de um asinino evidenciando o tendão interósseo (seta) e sua bifurcação (cabeça de seta) sobre a superfície abaxial dos ossos sesamóides proximais

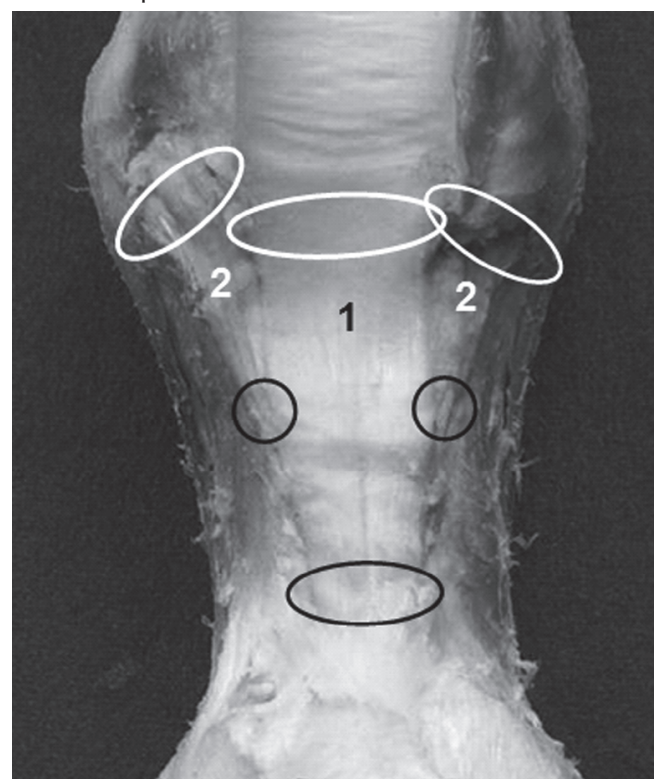

Figura 6 - Fotografia de dissecação da vista palmar do membro torácico de um asinino em dissecação profunda mostrando a inserção proximal (circulo branco) e distal (círculo preto) dos ligamentos sesamóideos reto (1) e oblíquos (2) sobre a superfície palmar da falange proximal e sob o terço médio do ligamento sesamóideo reto (Figura 6).

\section{Discussão}

A dissecação anatômica da superfície dorsal da região distal dos membros torácicos de asininos permitiu a distinção entre duas divisões clássicas: uma extensora e outra flexora que abrange em seu território grupos musculares distintos, como relatado para os eqüinos. ${ }^{5,6,7} \mathrm{O}$ músculo extensor digital comum foi evidenciado como o principal da divisão. Apresenta característica anatômica e disposição semelhante àquela que tem sido descrita na literatura para eqüinos ${ }^{8,9,10,11}$, apresentando um arranjo muscular com fibras que impossibilitam uma separação anatômica precisa entre as cabeças musculares. $\mathrm{O}$ tendão desse músculo surge de um ventre penado a uma distância um tanto menor do que o observado em eqüinos 12,13,14,15,16, contudo, obedecendo à mesma sintopia anatômica. Todavia, ao passar pela articulação metacarpofalangeana, emite um pequeno número de fibras tendíneas para a extremidade distal da falange, caracterizando uma inserção também nesse ponto, o que não foi descrito por estes autores. Em eqüinos esta região é usualmente acometida por traumas e contusões. ${ }^{17}$ Nos asininos, a presença de uma segunda inserção tendínea provavelmente surge como um mecanismo biomecânico adaptativo na prevenção de traumas regionais, promovendo, assim, uma maior resistência locomotora para esta espécie. ${ }^{18,19} \mathrm{O}$ tendão do músculo extensor digital lateral dos asininos apresenta-se da mesma maneira que o descrevem para eqüinos. 11,12,20,21,22,23 Contudo, nossas observações divergem das desses autores quanto ao seu ponto de inserção na articulação metacarpofalangeana, onde sofre um desvio em relação ao tendão extensor digital comum. Na divisão flexora, os músculos flexores digitais superficial e profundo apresentam características semelhantes àquelas descritas para 
eqüinos ${ }^{6,9,24,25}$, embora apresentem menor volume.

O ligamento interósseo apresenta inserção proximal semelhante ao observado em eqüinos. ${ }^{6,9,26,27,28}$ Porém as inserções distais de seus ramos extensores, em nossas observações, encontram o tendão do músculo extensor digital comum em um nível mais distal do que o descrito para eqüinos.

Os ligamentos observados à dissecação profunda da região distal dos membros de asininos apresentam características anatômicas diferenciadas. Os ligamentos sesamóideos retos e oblíquos de eqüinos são visualizados como bandas dispostas longitudinalmente sob a face palmar da falange média. ${ }^{29,30,31,32}$ Contudo, nossos achados divergem desses autores, quando evidenciamos que em asininos esses ligamentos mostram-se bem mais largos e bem mais definidos, particularmente, o sesamóideo reto, propiciando assim, um maior suporte para a sustentação e equilíbrio biodinâmico da articulação.

Os ligamentos palmares da articulação interfalangeana dos asininos apresentaram suas características anatômicas conforme o que foi descrito em eqüinos. ${ }^{33,34,35,36}$ Todavia, em nossas dissecações os ligamentos colateral lateral e medial da articulação interfalangeana proximal são observados como adotando uma disposição um tanto mais paralela aos ramos extensores do ligamento interósseo, o que se contrapõe aos achados destes autores.

\section{Conclusão}

- Os músculos flexores digitais superficial e profundo em asininos apresentam ventres musculares com um menor volume quando comparados aos eqüinos;

- Os tendões dos músculos flexores digitais superficial e profundo apresentam especializações adaptadas à sustentação do aparelho suspensório desses animais, caracterizados por uma maior área de inserção e espessura;

- Os ligamentos sesamóideos retos mostraram-se mais robustos e com uma maior área de inserção proximal palmar e lateralmente à base dos ossos sesamóides proximais, o que sugere algum tipo de especialização adaptada à sustentação do aparelho suspensório desses animais, mediante ao seu tipo de trabalho físico.

\section{Tendon and ligament anatomy of the distal forelimb region in donkey (Equus asinus) used to traction role and relations with the domestic equine anatomy}

\begin{abstract}
The asinine species play an essential role in the northeast region of Brazil in the income of the local families and consequently in the local economy. Due to a large number of locomotor disorders and a lack of professional care, an anatomical study of the distal forelimb region of the asinine was carried out in order to gather information to improve the clinical and surgical practice in this species, and to explain the less susceptibility to locomotor disorders compared to equines. The anatomical study showed that asinine has similar muscular characteristics already described for equines. Tendons and ligaments showed proper characteristics with different origin and attachment already described for equines, emitting tendons projections that improve articular insertion and better stability in its suspensory apparatus allowing a bigger safety during the movement in physic activities.
\end{abstract}

Key words: Asinines. Tendons. Joint ligaments. Phalanges. 


\section{Referências}

1 ROMASZKAN, G.; JUNQUEIRA, R. R. O cavalo. Itatiaia: Belo Horizonte, 1980. v. 1, p. 11.

2 TORRES, A. D.; JARDIM, W. R. Criação do cavalo e de outros eqüinos. São Paulo: Nobel, 1983. p. 270282 .

3 ASSOCIAÇÃO PARA O ESTUDO E PROTEÇÃO DO GADO ASININO. Raça Asinina das terras de Miranda: a origem da espécie e da raça. Disponível em: <www.aepga.pt>. Acesso em: 7 abr. 2004.

4 GOVERNO DA REPÚBLICA DE HUÍLA. O gado Asinino. Economia de tracção e de transporte no rural profundo. Um projeto no quadro da estratégia de desenvolvimento, v.10, p. 29-34, 2000. Disponível em: <www.huilanet.org > Acesso em: 7 abr. 2004.

5 BOLEN, G.; BUSONI, V.; JACQMOT, O.; SNAPS, F. Sonographic anatomy of the palmarodistal aspect of the equine digit. Radiology \& Ultrasound, v. 48, n. 3, p. 270-275, 2007.

6 SISSON, S. Músculos do eqüino. In. GETTY, R. Sisson/ Grossman - anatomia dos animais domésticos. 5. ed Rio de Janeiro: Guanabara Koogan, 1986. p. 395-402.

7 ZARUCCO, L.; WISNER, E. R.; SWANSTROM, M. D.; STOVER, S. M. Image fusion of computed tomographic and magnetic resonance images for the development of a three-dimensional musculoskeletal model of the equine forelimb. Veterinary Radiology $\boldsymbol{\&}$ Ultrasound, v. 47, n. 6, p. 553-562, 2006.

8 DYCE, K. M.; SACK, W. O.; WENSING, C. J. G. Tratado de anatomia veterinária. Rio de Janeiro: Guanabara Koogan, 1997a. p. 227-336.

9 PASIN, M.; BRASS, K. E.; ROSAURO, A. C.; OLIVEIRA, F. G.; FIGUEIRÓ, G. M.; FIALHO, S. S.; SILVA, C. A. M. Caracterização ultra-sonográfica dos tendões flexores em eqüinos: região metacarpiana ultrasonographic characterization of the flexor tendons in horses: metacarpal region. Arquivos da Faculdade de Veterinária, v. 29, n. 2, p.131-138, 2001.

10 SAMPSON, R. T. Magnetic Resonance Imaging of the Proximal Metacarpal and Metatarsal Regions. Clinical Techniques in Equine Practice, v. 6, n. 1, p. 78-85, 2007.

11 MURRAY, R. C. Magnetic Resonance Imaging of the Equine Carpus. Clinical Techniques in Equine Practice, v. 6, p. 86-95, 2007.

12 TOMLINSON, J. E.; REDDING, W. R.; BERRY C.; SMALLWOOD, J. E. Computed tomographic anatomy of the equine tarsus. Veterinary Radiology $\boldsymbol{\&}$ Ultrasound, v. 44, n. 2, p.174-178, 2003.

$13 \mathrm{KÖNIG}, \mathrm{H}$. E. Anatomia dos animais domésticos: texto e atlas colorido. Porto Alegre: Artmed, 2002. v. 1, p. $165-201$.

14 BARNABÉ, P. A.; CATTELAN, J. W.; CADIOLI, F.
A. Anatomofisiologia da bainha tendínea sinovial digital dos eqüinos. Ciência Rural, v. 34, p. 619-623, 2004.

15 MCGHEE, J. D.; WHITE, N. A.; GOODRICH, L. R. Primary desmitis of the palmar and plantar annular ligaments in horses: 25 cases. Journal of the American Veterinary Medical Association, v. 226, n. 1, p. 83-86. 2005.

16 BUSONI, V.; HEIMANN, M.; TRENTESEAUX, J.; SNAPS, F.; DONDELINGER, R. F.Magnetic resonance imaging findings in the equine deep digital flexor tendon and distal sesamoid bone in advanced navicular diseasean ex vivo study. Veterinary Radiology \& Ultrasound, v. 46, n. 4, p. 279-286, 2005.

17 MARR, C. M.; MCMILLAN, I.; BOYD, J. S. WRIGHT, N. G.; MURRAY, M. Ultrasonographic and histopathological findings in equine superficial digital flexor tendon injury. Equine Veterinary Journal, v. 25, n. 1, p. 23-29, 1993.

18 ALVES, F. R.; GUERRA, P. C.; MARIANA, A. N. B.; VULCANO, L. C. Avaliação clínica e radiográfica de alterações ósseas da região distal dos membros torácicos de asininos(Equus asinus) utilizados na tração animal no município de São Luís-MA. Revista de educação continuada do CRMV-SP, v. 6, n. 1/3, p. 42-52, 2003.

19 ALVES, F. R.; MARIANA, A. N. B.; GUERRA, P. C.; MACHADO, P. P.; CRUZ-PINTO, C. E. Avaliação anatômica da secção da área transversal dos tendões da região distal dos membros torácicos de asininos (Equus asinus) utilizados como veículo de tração. Revista de educação continuada do CRMV-SP, v. 8, n. 2, p. 145155, 2005.

20 KASASHIMA, Y.; SMITH, R. K.; BIRCH, H. L.; TAKAHASHI, T.; KUSANO, K.; GOODSHIP, A. E. Exercise-induced tendon hypertrophy: cross-sectional area changes during growth are influenced by exercise. Equine Veterinary Journal, v. 34, p. 264-268, 2002. Supplement.

21 GREIG, C. R.; BRASS, K. E.; OLIVEIRA, F. G.; DE LA CORTE, F. D.; SILVA, J. H.; SILVA, C. A. M. Relevância da ultra-sonografia dos tendões flexores em cavalos Puro Sangue de corrida na adaptação ao treinamento. Ciência Rural, v. 35, n. 4, p. 832-836, 2005.

22 SCHALLER, C. G. M.; HABEL, R. E.; SACK, W. O.; SIMOENS, P.; VOS, N. R. Nomenclatura anatômica ilustrada: angiologia. São Paulo: Manole, 1999. p. 282-302.

23 SMALLWOOD, J. A. A guide tour of veterinary anatomy: domestic ungulates and laboratory mammals. Philadelphia: W. B. Saunders, 1992. p. 271-287.

24 CONSTANTINESCU, G. M. Clinical dissection guide for large animals: horse, ox, sheep, goat, pig. Saint Louis: Mosby - Year Book, 1991. p. 230-258.

25 NICKEL, R.; SCHUMMMER, A.; SEIFERLE, E. The locomotor system of the doméstic mammals. Berlin Hamburg: Paul Parey, 1986. v. 3, p. 197-200. 
26 PICKERSGILL, C. H.; MARR, C. M.; REID, S. W. J. Repeatability of diagnostic ultrasonography in the assessment of the equine superficial digital flexor tendon. Equine Veterinary Journal, v. 33, n. 1, p. 33-37, 2001

27 TNIBAR, M.; KASER-HOTZ, B.; AUER, J. A. Ultrasonography of the dorsal and lateral aspects of the equine carpus: technique and normal appearance. Veterinary Radiology \& Ultrasound, v. 34, n. 6, p. 413-425, 1993.

28 LATORRE, R.; ARENCIBIA, A.; GIL, F.; RIVERO, M.; HENRY, R. W.; RAMÍREZ, G.; VÁQUEZ, J. M. Correlation of magnetic resonance images with anatomic features of the equine tarsus. American Journal of Veterinary Research, v. 67, n. 5, p. 756-761, 2006

29 BUSONDI, V.; SNAPS, F. Effect of deep digital flexor tendon orientation on magnetic resonance imaging signal intensity in isolated equine limbs-the magic angle effect. Veterinary Radiology \& Ultrasound, v. 43, n. 5, p. 428-430, 2002.

30 ZUBROD, C. J.; FARNSWORTH, K. D.; TUCKER, R. L.; RAGLE, C. A. Injury of the collateral ligaments of the distal interphalangeal joint diagnosed by magnetic resonance. Veterinary Radiology \& Ultrasound, v. 46, n. 1 , p. 11-16, 2005

31 SAMPSON, S. N.; SCHNEIDER, R. K.; TUCKER, R. L.; GAVIN, P. R.; ZUBROD, C. J.; HO, C. P. Magnetic resonance imaging features of oblique and straight distal sesamoidean desmitis in 27 horses. Veterinary Radiology \& Ultrasound, v. 48, n. 4, p. 303-311, 2007.

32 VAN SCHIE, H. T.; BAKKER, E. M.; JONKER, A.; WEEREN, P. R. Ultrasonographic tissue characterization of equine superficial digital flexor tendons by means of gray level statistics. American Journal of Veterinary Research, v. 61, n. 2, p. 210-219, 2000.

33 MURRAY, R. C.; ROBERTS, B. L.; SCHRAMME, M. C.; DYSON, S. J.; BRANCH, M. Quantitative evaluation of equine deep digital flexor tendon morphology using magnetic resonance imaging. Veterinary Radiology \& Ultrasound, v. 45, n. 2, p. 103-111, 2004.

34 BLAIK, M. A.; HANSON, R. R.; KINCAID, S. A.; HATHCOCK, J. T.; HUDSON, J. A.; BAIRD, D. K. Low-field magnetic resonance imaging of the equine tarsus: normal anatomy. Veterinary Radiology \& Ultrasound, v. 41, n. 2, p. 131-141, 2000.

35. SPRIET M.; MAI, W.; MCKNIGHT, A. Asymmetric signal intensity in normal collateral ligaments of the distal interphalangeal joint in horses with a low-field MRI system due to the magic angle effect. Veterinary Radiology \& Ultrasound, v. 48, n. 2, p. 95100, 2007.

36 MALONE, E. D.; LES, C. M.; TURNER, T. A. Severe Carpometacarpal Osteoarthritis in Older Arabian Horses.Veterinary Surgery, v. 32, n. 3, p. 191-195, 2003. 